\title{
Are high-lysine cereal crops still a challenge?
}

R.R. Ferreira ${ }^{1}$, V.A. Varisi ${ }^{2}$ L.W. Meinhardt², P.J. Lea ${ }^{3}$ and R.A. Azevedo ${ }^{2}$
1Departamento de Genética e Evolução, Instituto de Biologia, Universidade Estadual de Campinas, Campinas, SP, Brasil

2Departamento de Genética, Escola Superior de Agricultura "Luiz de Queiroz", Universidade de São Paulo, Piracicaba, SP, Brasil

${ }^{3}$ Department of Biological Sciences, Lancaster University, Lancaster, LA1 4YQ, UK

\section{Correspondence}

R.A. Azevedo

Departamento de Genética

ESALQ, USP

Caixa Postal 83

13400-970 Piracicaba, SP

Brasil

Fax: +55-19-3433-6706

E-mail: raazeved@esalq.usp.br

Research supported by FAPESP and the British Council.

.....................

Received November 26, 2004 Accepted April 18, 2005

......................

\section{Abstract}

The essential amino acids lysine and threonine are synthesized in higher plants via a pathway starting with aspartate that also leads to the formation of methionine and isoleucine. Lysine is one of most limiting amino acids in plants consumed by humans and livestock. Recent genetic, molecular, and biochemical evidence suggests that lysine synthesis and catabolism are regulated by complex mechanisms. Early kinetic studies utilizing mutants and transgenic plants that overaccumulate lysine have indicated that the major step for the regulation of lysine biosynthesis is at the enzyme dihydrodipicolinate synthase. Despite this tight regulation, recent strong evidence indicates that lysine catabolism is also subject to control, particularly in cereal seeds. The challenge of producing crops with a high-lysine concentration in the seeds appeared to be in sight a few years ago. However, apart from the quality protein maize lines currently commercially available, the release of high-lysine crops has not yet occurred. We are left with the question, is the production of high-lysine crops still a challenge?

\section{Hunger and malnutrition}

Hunger and malnutrition are among the most devastating problems affecting a large part of the world's population. Nearly $30 \%$ of people in the developing world are suffering from one or more forms of malnutrition (1). In fact, one billion people worldwide are affected by nutritional deficiencies, 800 million people are chronically undernourished, more than $40 \%$ of women in the developing world are underweight and/or anemic, and one third of the world's children are affected
Key words

- Lysine

- Threonine

- Aspartate

- Nutritionally improved crops

- Methionine

- Amino acids

.... Amino acids by delayed growth and development caused by malnutrition (2). Furthermore, vitamin A deficiency worldwide on a yearly basis causes blindness in around 500,000 children and eye damage in more than 14 million people (2).

The tragic consequences of malnutrition include disability, stunted mental and physical growth and death, affecting all age groups. In the embryo and fetus, nutritional disorders are responsible for low birth weight, brain damage and neural tube defects (2). Low birth weights are linked to malnutrition 
of the mothers before and during pregnancy, while micronutrient deficiencies in mothers have been shown to increase the mortality of young children. Furthermore, malnourished children are less able to fight infections (3). In the infant and adolescent stages of life, protein-energy malnutrition and vitamin A deficiencies are linked to developmental and growth retardation, increased risk of infection and higher risks of death and blindness $(1,2)$. In the elderly, protein-energy malnutrition is the most important deficiency resulting in a higher risk of death, increased risk of infection and a reduced quality of life (1).

Overall, progress during the last twenty years in reducing protein-energy malnourishment among infants and young children has been exceedingly slow, while micronutrient and vitamin deficiency have contributed to worsen the situation (1).

\section{Protein deficiency}

Protein quality is based on their amino acid composition (particularly their relative content of essential amino acids) and their digestibility. Therefore, high quality proteins are those that are easily digested and contain the essential amino acids in quantities that correspond to human requirements $(1,2)$. Amino acid and protein requirements are a function of the metabolic demands of the organism and the efficiency with which they can be utilized to meet these demands (4).

Early studies with animals have shown that threonine, sulfur containing amino acids and isoleucine are the most important amino acids for young rats (5), whereas isoleucine and tryptophan are important for adult rats (6) and sulfur amino acids, threonine and tryptophan are important for adult pigs (7). Both leucine and lysine are the most abundant amino acids in carcass proteins and in the growth requirements for rats and pigs $(5,7)$.
In humans, early reports suggested that the human protein needs are around $180 \mathrm{~g} /$ day. However, studies with lower protein diets resulted in values of $50 \mathrm{~g} / \mathrm{day}$. The latter value is similar to the one currently accepted by FAO/WHO (4). However, ElKhoury et al. (8) suggest that the FAO/ WHO/UNU lysine requirement value is not sufficient to maintain lysine homeostasis in healthy adults and, based on their results and tracer studies done by others, the mean lysine requirement of healthy adults was determined to be $30 \mathrm{mg} \mathrm{kg}^{-1}$ day $^{-1}$. In addition, the results of two-phase linear regression crossover analysis showed that the mean threonine requirement was $19.0 \mathrm{mg} \mathrm{kg}^{-1}$ day $^{-1}$ with an upper safe intake of $26.2 \mathrm{mg} \mathrm{kg}^{-1}$ day $^{-1}(9)$.

\section{Amino acids}

Amino acids are the main nitrogen storage compounds in plants and the basis for the synthesis of proteins (10). Twenty different amino acids are usually incorporated into proteins (11), although they can be subject to post-translational modifications such as phosphorylation, hydroxylation, methylation, and acetylation. Besides those that compose proteins, more than 300 additional amino acids have been characterized in plants (12). These non-protein amino acids can play a vital role as metabolic pathway intermediates, such as ornithine, homoserine and cystathionine. In addition, they can also act as nitrogen storage molecules, as in the case of canavanine, or they can be synthesized in response to stress, revealing the possibility that some of them may act as insecticidal and fungicidal agents (11).

Humans and monogastric animals are not able to synthesize nine of the amino acids that are found in proteins. These nine amino acids (lysine, threonine, methionine, phenylalanine, tryptophan, isoleucine, leucine, valine, and histidine) are designated essential amino acids and must be acquired 
through the diet (13). In addition, animals are only able to synthesize tyrosine and cysteine by conversion from phenylalanine and methionine, respectively. Plants and most of the bacteria and fungi have the capacity to synthesize all the twenty amino acids necessary for protein synthesis (11), which can be classified into "families" according to the precursor of the biosynthetic pathway. These pathways are subjected to complex regulation so that there is limited waste of energy and key carbon, nitrogen and sulfur substrates. Early cell fractionation and more recent gene sequencing studies have clearly shown that the enzymes involved in the synthesis of essential amino acids are located in the chloroplasts of the leaves, or in the plastids of non-photosynthetic organs, such as seeds and roots (11).

Recent research has revealed that plant proteins supply $65 \%$ of the total global ingested proteins, with cereal grains representing $47 \%$. Plant proteins in developed countries constitute a low proportion compared with animal sources. However, in the developing countries plant proteins are the main and, in many cases, the only source of protein (14). Despite their importance, cereals are usually deficient in lysine, threonine and tryptophan, while legumes are deficient in methionine. These nutritional deficiencies have given rise to a great deal of interest in research into the metabolic pathway from aspartate to lysine, threonine, methionine and more recently the conversion of threonine to isoleucine, which is a key target of a number of potent herbicides (15).

\section{The aspartate metabolic pathway}

Aspartate is able to act as a precursor for two main pathways. The first leads to the synthesis of asparagine, which is one of the main compounds utilized for the transport and storage of nitrogen in plants. Asparagine is synthesized by the transfer of the nitrogen from the amide group of glutamine to aspartate, catalyzed by asparagine synthetase. The enzyme asparaginase carries out the catabolism of asparagine forming ammonia, which is reassimilated through the glutamate synthase cycle (16).

Aspartate is also the precursor of the aspartate "family" of amino acids, lysine, threonine, methionine, and isoleucine $(15,17$, 18) (Figure 1). Due to the low lysine and threonine concentrations in cereal seeds and their importance as essential amino acids, studies have been carried out in order to obtain a better understanding of the regulation of the pathway, with the ultimate aim of constructing plants by genetic manipulation that can overproduce and accumulate higher amounts of lysine and threonine in the seeds $(15,19-24)$.

Aspartate kinase (AK, EC 2.7.2.4), the first enzyme of the pathway, can be present as several isoenzymes, which are feedback inhibited either by lysine or by threonine. AK catalyzes the phosphorylation of aspartate to $B$-aspartyl phosphate, which is then converted to ß-aspartyl semialdehyde (ASA) in a reaction catalyzed by the enzyme aspar-

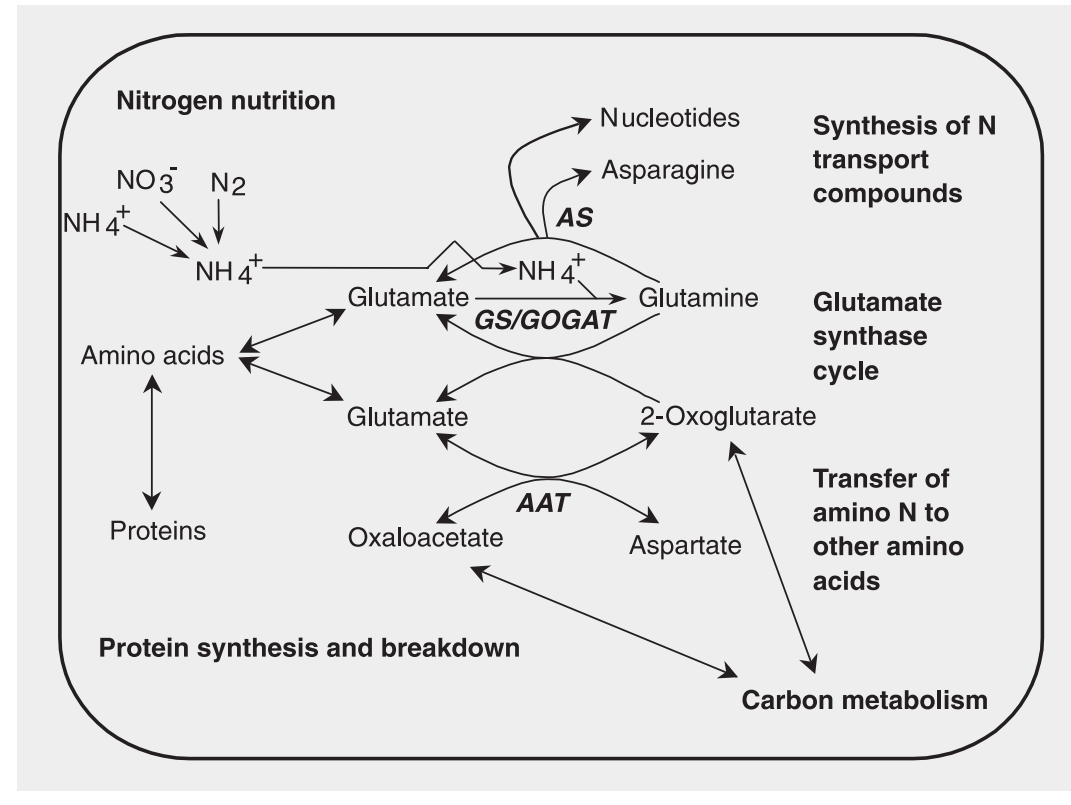

Figure 1. Enzyme reactions important in the assimilation of ammonia. GS = glutamine synthetase; GOGAT = glutamate synthase; $A S=$ asparagine synthetase; AAT = aspartate aminotransferase. 
tate semialdehyde dehydrogenase (EC 1.2.1. 11). At this point, the pathway is divided into two branches, one leading to lysine formation, whereas the other branch is subsequently divided to form two sub-branches, with one leading to the synthesis of threonine and the other to the synthesis of methionine (15).

The amino acid lysine is synthesized from ASA by seven consecutive enzymatic reactions initiated by the action of dihydrodipicolinate synthase (DHDPS, EC 4.2.1.52), which is strongly feedback inhibited by lysine (15). In the other branch, ASA is reduced to homoserine by a reaction catalyzed by homoserine dehydrogenase (HSDH, EC 1.1.1.3), the first enzyme committed to the synthesis of threonine and methionine, which also exists as separate isoenzymes, resistant and sensitive to threonine feedback inhibition (21). Homoserine is phosphorylated to

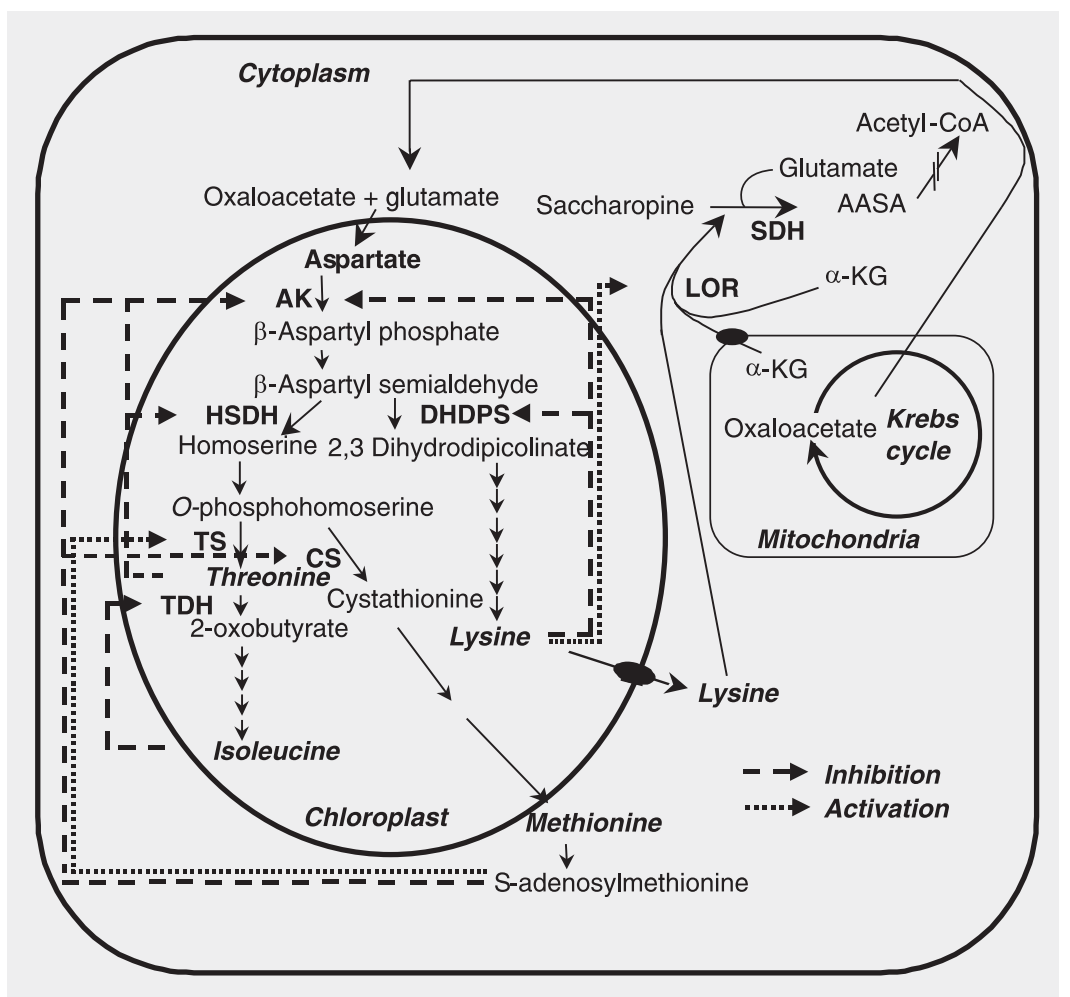

Figure 2. The aspartate metabolic pathway of higher plants. $\mathrm{AK}=$ aspartate kinase; $\mathrm{HSDH}=$ homoserine dehydrogenase; DHDPS = dihydrodipicolinate synthase; TS = threonine synthase; $\mathrm{CS}=$ cystathionine- $\gamma$-synthase $; \mathrm{TDH}=$ threonine dehydratase; LOR = lysine-2oxoglutarate reductase; $\mathrm{SDH}=$ saccharopine dehydrogenase; $\mathrm{AASA}=\alpha$-aminoadipic acid; $\alpha-K G=\alpha$-ketoglutarate.
$O$-phosphohomoserine by the action of the enzyme homoserine kinase (EC 2.7.1.39) and converted to threonine by threonine synthase (EC 4.2.99.2) (25). Isoleucine is synthesized directly from threonine by a series of five enzymatic steps (15). The synthesis of the amino acid methionine follows a separate branch of the pathway, which is initiated from $O$-phosphohomoserine and follows three enzymatic reactions involving the enzymes cystathionine $\gamma$-synthase (EC 4.2.99.9), cystathionine B-lyase (EC 4.4.1.8) and methionine synthase (EC 2.1.1.13) (21). $\mathrm{S}$-adenosylmethionine, which is one of the main methyl group donors in plants, is synthesized from methionine in an enzymatic reaction catalyzed by the enzyme S-adenosylmethionine synthase (EC 2.5.1.6) (26) (Figure 2). For a full detailed review of the aspartate metabolic pathway, see Ref. 15.

\section{The key enzymes involved in lysine metabolism}

AK was initially studied in bacteria and later characterized in detail in several plant species. At least two distinct AK isoenzymes have normally been observed in plants, threonine-sensitive and lysine-sensitive isoenzymes (15). The lysine-sensitive AK isoenzyme is a monofunctional polypeptide, whereas the threonine-sensitive $\mathrm{AK}$ isoenzyme is a bifunctional polypeptide containing a threonine-sensitive HSDH domain $(15,27)$. The distribution of the AK isoenzymes may vary depending on the tissue and developmental stage; however, the lysinesensitive AK isoenzyme normally accounts for the majority of AK activity (15). A controversial regulation of AK activity by calcium, a modulator of the activity of several enzymes and an important messenger in signal transduction in plants, and by calmodulin, a key component of a complex cascade system of protein kinases, has also been reported in the literature (15). The threonine-resistant and threonine-sensitive HSDH 
isoenzymes have also been studied in detail and purified from plant species (17). Purification of AK and HSDH isoenzymes by ionexchange and gel filtration chromatography has produced the best results and the molecular mass of the isoenzymes has been shown to vary from 104 to $250 \mathrm{kDa}$ for $\mathrm{AK}$ and from 70 to $190 \mathrm{kDa}$ for HSDH (15). The activity of the threonine-sensitive HSDH isoenzyme can be easily altered by $\mathrm{KCl}$ and the isoenzyme is involved in the biosynthesis of amino acids in the plastids, while the physiological function of the cytoplasmic threonine-resistant HSDH isoenzyme is still unknown (15).

DHDPS, the first enzyme directly involved in lysine biosynthesis, is strongly regulated by feedback inhibition by low concentrations of this amino acid and consequently plays the most important role in the regulation of lysine biosynthesis $(17,28,29)$. DHDPS catalyzes the condensation of pyruvate and aspartate semialdehyde to dihydrodipicolinic acid, and has been purified and characterized in plants, exhibiting a molecular mass of 115 to $167 \mathrm{kDa}(15,17)$.

\section{Lysine catabolic pathway}

Due to the need of obtaining cereal seeds with a higher lysine concentration, the lysine catabolic pathway (Figure 2) has also been studied recently in considerable detail. Initial studies of lysine catabolism in plants were carried out using ${ }^{14} \mathrm{C}$-labeled lysine, with radioactivity being incorporated into glutamate and $\alpha$-aminoadipic acid (30). Lysine degradation has been shown to be catalyzed by two main enzymes, lysine 2oxoglutarate reductase (LOR), also known as lysine $\alpha$-ketoglutarate reductase, which catalyzes the formation of saccharopine, and saccharopine dehydrogenase (SDH), which hydrolyzes saccharopine to glutamate and $\alpha$-aminoadipic acid (31). Most of the LORSDH activities are present in a single bifunctional polypeptide $(31,32)$, although mono- functional forms of both enzymes have also been detected (33). Recent studies have indicated that LOR-SDH-encoding genes are expressed in several tissues, particularly during osmotic stress (34). The biochemical characterization of the bifunctional enzyme LOR-SDH has revealed that LOR activity is stimulated by lysine via a complex cascade of calcium-dependent phosphorylation (35).

Perhaps the most significant finding concerning lysine catabolism has been that related to the opaque- 2 maize mutant. This mutant is characterized by a high-lysine concentration when compared to wild-type maize seeds, a property due to altered storage protein distribution and higher accumulation of lysine in the soluble form (36). Biochemical and molecular analyses have revealed that the $o 2$ gene, which encodes a transcriptional activator factor, regulates the LOR activity in the maize endosperm. In the opaque-2 mutant, the LOR enzymatic activity, protein content and mRNAs have been shown to be reduced according to changes in expression pattern during the development of the grain $(37,38)$. In addition, elevated lysine concentrations were detected in mature seeds in a knockout mutant of Arabidopsis thaliana which eliminated expression of the gene encoding the bifunctional LOR-SDH enzyme (24).

\section{High-lysine mutants and transgenic plants}

The need to increase the nutritional content of cultivated plant has been recognized for a long time. Perhaps one of the most important advances in recent years was the development of "golden rice", a transgenic line produced by expressing in the rice endosperm the synthesis pathway of $B$-carotene, the precursor of vitamin A in the human body (39). Such $B$-carotene-rich seeds may be able to alleviate vitamin A deficiency and elite lines of rice should soon be available to farmers in the developing coun- 
tries at a low cost (40).

For the production of plants with high lysine concentrations, several strategies have been implemented. The conventional longterm plant breeding programs, although limited, have been used to select plants with improved protein quality (41). Naturally occurring high-lysine mutants such as the opaque- 2 and floury maize mutant variations are excellent examples of the success of such a simple and traditional, but effective approach. Unfortunately the high-lysine mutants exhibited undesirable agronomic characteristics (36), which only more recently have been partially overcome with the development of quality protein maize (QPM) (36). Biochemical mutants have been isolated that exhibit altered regulatory patterns for the specific targeted enzymes, such as AK and DHDPS, which were less sensitive or insensitive to feedback inhibition by their end product amino acids or their analogues (18). Finally, in the 1990's, a range of transgenic plants were produced, in which key enzymes had been genetically manipulated with the aim of producing high-lysine plants $(28,42,43)$.

The last two strategies described were essential for the understanding of the regulation of the amino acids synthesized through the aspartate metabolic pathway. The knowledge accumulated from the initial work on microorganisms, later associated with studies conducted on legume crops, were also critical for the determination of the fundamental aspects of lysine metabolism. It has been proposed that lysine overproduction can be obtained by the alteration of the sensitivity of DHDPS to this amino acid, which would result in an increased rate of lysine synthesis. Nonetheless, it may also be important to reduce the lysine-feedback inhibition of the lysine-sensitive AK isoenzyme. However, the manipulation of lysine biosynthesis did not ensure lysine accumulation in the cereal seeds, but did so in the leaves (17). Therefore, the accumulation of lysine in the seeds appears to be dependent on the manipulation of the rate of catabolism. This statement is further supported by the fact that LOR and SDH activities are very high in the endosperm of cereal seeds, but are hardly detectable in other tissues. In addition, the high-lysine maize opaque-2 mutant was shown to contain very low LOR and SDH activities, which reduced the degradation of lysine and allowed a higher amount of the amino acid to be incorporated into the storage proteins, with the excess lysine being accumulated in the soluble form in the endosperm. The decrease in the LOR and SDH activities is due to a reduction in the transcription of the ZLKRSDH gene and thus in the amount of LOR-SDH protein (37).

The data obtained from legume plants, which normally contain much higher lysine concentrations in the grain, are also particularly relevant and add further support to the important role played by LOR and SDH in lysine degradation. Intermediate compounds of lysine catabolism accumulated in the seeds of soybean and canola plants (43), suggesting a reduced activity of LOR and SDH in these species which naturally exhibit much higher lysine contents when compared to those of the cereal crops (43). Similarly, the LOR and SDH activities of Phaseolus vulgaris seeds and even some other tissues were also shown to be drastically reduced compared to cereals $(44,45)$. Even among cereal crops, rice exhibits a very different storage protein profile and a naturally higher soluble lysine and protein concentration, suggesting a different rate of lysine metabolism as confirmed by the lower LOR-SDH activities observed in the grain (32).

Unlike lysine, overproduction and accumulation of threonine have been frequently obtained following the alteration of the feedback inhibition of the lysine-sensitive AK $(18,46)$. Threonine accumulates in all tissues, including seeds, suggesting that enzymes of threonine catabolism are not active 
in plants, as compared to those of lysine catabolism (18).

Another aspect concerning the accumulation of lysine is the participation of amino acid transporters, since a significant concentration of amino acids is transported from distant plant tissues to the developing seeds through the phloem. Phloem unloading in the seed coats is considered to be symplasmic. The symplasmic discontinuity between maternal and filial tissues in seeds necessitates membrane efflux from the maternal tissues and subsequent uptake by filial tissues such as the endosperm or embryo.

More than 50 distinct amino acid transporter genes have been identified in the genome of Arabidopsis thaliana, indicating that transport of amino acids across membranes is a highly complex feature in plants $(47,48)$. Lysine has been shown to be transported by two members of a family of broad specificity transporters (AAP). AAP 5 was expressed in mature leaves and stems, indicating a possible role in phloem loading (49), while AAP 3 was expressed only in the roots. More recently a family of nine cationic amino acid transporters (CAT) has been characterized from $A$. thaliana, with CAT 1 and CAT 5 exhibiting a high affinity for arginine and lysine (48). CAT 1 was specifically expressed in the major veins of leaves and roots, in various floral tissues and the developing seed (50), while CAT 5 was expressed in cotyledons during early seedling development and at the edges of leaves (48).

Thus, there still is little information about lysine transport to the seeds. Analysis of amino acid composition of the vascular sap of the opaque-2 high-lysine maize mutant was determined during kernel development revealing that glutamine was the major amino acid transported to the endosperm (51). Perhaps the first clear evidence that the rate of lysine breakdown appeared to be an important mechanism by which the opaque- 2 maize high-lysine mutant controls lysine level in maize endosperm was obtained when the lysine content in the sap was compared with the lysine stored in both wild-type and sugary opaque- 2 maize endosperm during kernel filling (52). The lysine content of the ear peduncle sap could account for all the lysine found in both endosperms. Preformed lysine was shown to be highly catabolized in the wild-type endosperm, but not in the highlysine sugary opaque-2 endosperm (52).

\section{Opaque-2 and quality protein maize}

The opaque-2 maize mutant exhibits a high seed lysine content, but a small amount when compared to wild-type maize plants (36). This mutation confers an opaque phenotype to the maize grain and a floury endosperm (36). In addition to the high-lysine and high-tryptophan traits, several other characteristics have also been shown to be altered in the opaque- 2 mutant, including the activity of RNase and of enzymes of carbon and nitrogen metabolism (36), a reduction in protandry, and the differences in photosynthetic activity of young seedlings (53). A range of genes belonging to several distinct metabolic pathways, such as glycolysis, amino acid biosynthesis and storage proteins, were shown to be direct or indirect targets of the $o 2$ gene product (31). Molecular and biochemical analyses also confirmed that the $o 2$ gene regulates LOR activity in the maize endosperm (31). An opaque-2 box was found in the 5' regulatory region, which confirmed the transcriptional regulation of the LOR-SDH-encoding gene by the $o 2$ gene product (31). Proteome and transcriptome analyses of the regulatory role of the $o 2$ gene found one restriction site on the 3 ' side of the $o 2$ gene, which is associated with the amount of LOR-SDH mRNAs (31).

Despite the modification in seed structure and nutritional quality, the opaque- 2 plants exhibited a reduced productivity and a higher susceptibility to pathogens, which was a major commercial drawback. The cor- 
relation between nutritional quality and yield has been a serious issue over the years, since the two factors appear to be negatively correlated. This problem appears now to have been overcome since the introduction of modifier genes ( $m o 2$ genes) that changed the opaque-2 phenotype of the seed, thus allowing wild-type-like seed characteristics to be maintained, resulting in normal yield but conserving the high lysine and high tryptophan concentrations (36). These new maize lines have been designated QPM and several hybrids were produced and introduced into the market. However, the widespread use of these varieties has not been as fast as initially expected. QPM varieties have also exhibited lower seed LOR and SDH activities when compared to the wild type, as would be expected considering their opaque- 2 origin (36). The effect of the $o 2$ gene is not restricted to lysine catabolism, but also appears to regulate the biosynthesis of this amino acid, since the analysis of a maize double mutant opaque-2/ask1 (gene encoding the lysine-sensitive AK), indicated alterations in total and soluble amino acids, storage proteins and enzymatic activity, suggesting that the askl gene may be regulated by $o 2$ (54). In addition, genetic mapping analyses have linked the askl gene to the $o 2$ gene (46). Other evidence of the regulatory role of $o 2$ regarding the AK isoenzymes was later obtained from quantitative trait loci analysis (55).

While the value of the opaque-2 maize mutant in improving our understanding of lysine metabolism is clear, several other recessive and semi-dominant high-lysine maize mutations have been available for some time, but only recently have some of them been investigated concerning lysine metabolism and storage proteins (56-58). Transcriptomic and proteomic approaches are now available and are powerful tools that can be used in the study of these mutants, which should allow further insights to be gained into the complex regulatory network of the $o 2$ gene and product. Will these mutants (e.g. opaque and floury maize mutants) behave like the opaque-2 mutant? Are there alternative mutants that could be more useful than the opaque-2 mutant in terms of commercial use?

These questions are also valid for other cereal crops such as rice, barley and sorghum, for which high-lysine mutants (e.g., high-lysine sorghum mutants, hiproly barley mutant, high-lysine rice mutants) have been available for some time $(18,59,60)$. Very little is known about the aspartate metabolic pathway and, more specifically, lysine metabolism in the mutants of crops other than maize. Initial results with a range of high-lysine maize mutants have already revealed important differences among the mutants in relation to the wild types and the maize opaque-2 mutant (56-58). Interestingly, some high-lysine mutants have shown similar rates of lysine catabolism when compared to their wild-type lines, suggesting that a reduced lysine catabolic rate does not explain the increase in the levels of soluble lysine in the endosperm of these mutants (56-58).

In 1997 Azevedo et al. (15), when reviewing the aspartate metabolic pathway, hoped that in five years high-lysine transgenic crop plants would be available to farmers. Apart from the QPM lines, it would appear that very little else in the way of highlysine crops is available. Perhaps recent legislation and general concern about the use of modified genetic organisms have been the major setback regarding the release of such crops. 


\section{References}

1. WHO/NDH (2000). Nutrition for Health and Development. A global agenda for combating malnutrition, WHO, France.

2. WHO/PSF/FAP (1997). Food Aid for Health and Development. Food Aid Programs Unit, WHO, France.

3. Wagstaff A, Bustreo F, Bryce J \& Claeson M (2004). WHO; Poverty Working Group. Child health: reaching the poor. American Journal of Public Health, 94: 726-736.

4. Millward DJ (1998). Metabolic demands for amino acids and the human requirements: Millward and Rivers revisited (1988). Journal of Nutrition, 128: 2563s-2576s.

5. Gahl MJ, Finke MD, Crenshaw TD \& Benevenga NJ (1991). Use of a four-parameter logistic equation to evaluate the response of growing rats to ten levels of each indispensable amino acid. Journal of Nutrition, 121: 1720-1729.

6. Said A \& Hegsted DM (1970). Response of adult rats to low dietary levels of essential amino acids. Journal of Nutrition, 100: 13631376.

7. Fuller MF, McWilliam R, Wang TC \& Giles LR (1989). The optimum dietary amino acid pattern for growing pigs; requirements for maintenance and for tissue protein accretion. Brazilian Journal of Nutrition, 62: 255-267.

8. El-Khoury AE, Pereira PCM, Borgonha S, Basile-Filho A, Beaumier L, Wang SY, Metges CC, Ajami AM \& Young VR (2000). Twentyfour-hour oral tracer studies with $\mathrm{L}-\left[1^{-13} \mathrm{C}\right] \mathrm{lysine}$ at a low $(15 \mathrm{mg}$ $\left.\mathrm{kg}^{-1} \mathrm{~d}^{-1}\right)$ and intermediate $\left(29 \mathrm{mg} \mathrm{kg}^{-1} \mathrm{~d}^{-1}\right)$ lysine intake in healthy adults. American Journal of Clinical Nutrition, 72: 122-130.

9. Wilson DC, Rafii M, Ball RO \& Pencharz PB (2000). Threonine requirement of young men determined by indicator amino acid oxidation with use of L-[ $\left.1^{-13} \mathrm{C}\right]$ phenylalanine. American Journal of Clinical Nutrition, 71: 757-764.

10. Medici LO, Azevedo RA, Smith RJ \& Lea PJ (2004). The influence of nitrogen supply on antioxidant enzymes in plant roots. Functional Plant Biology, 31: 1-9.

11. Lea PJ \& Azevedo RA (2003). Primary products: plant amino acids. In: Thomas B, Murphy SJ \& Murray BG (Editors), Encyclopaedia of Applied Plant Sciences. Vol. 3. Elsevier, Amsterdam, The Netherlands, 871-883.

12. Wink M (2003). Evolution of secondary metabolites from an ecological and molecular phylogenetic perspective. Phytochemistry, 64: 319.

13. Galili G, Galili S, Lewinsohn E \& Tadmor Y (2002). Genetic, molecular, and genomic approaches to improve the value of plant foods and feeds. Critical Reviews in Plant Sciences, 21: 167-204.

14. Millward DJ (1999). The nutritional value of plant-based diets in relation to human amino acid and protein requirements. Proceedings of the Nutrition Society, 58: 249-260.

15. Azevedo RA, Arruda P, Turner WL \& Lea PJ (1997). The biosynthesis and metabolism of the aspartate derived amino acids in higher plants. Phytochemistry, 46: 395-419.

16. Andrews M, Lea PJ, Raven JA \& Lindsey K (2004). Can genetic manipulation of plant nitrogen assimilation enzymes result in increased crop yield and greater $\mathrm{N}$-use efficiency? An assessment. Annals of Applied Biology, 145: 25-40.

17. Azevedo RA \& Lea PJ (2001). Lysine metabolism in higher plants. Amino Acids, 20: 261-279.

18. Azevedo RA (2002). Analysis of the aspartic acid metabolic pathway using mutant genes. Amino Acids, 22: 217-230.

19. Craciun A, Jacobs M \& Vauterin M (2000). Arabidopsis loss-of- function mutant in the lysine pathway points out complex regulation mechanisms. FEBS Letters, 487: 234-238.

20. Hesse H, Kreft O, Maimann S, Zeh M \& Hoefgen R (2004). Current understanding of the regulation of methionine biosynthesis in plants. Journal of Experimental Botany, 55: 1799-1808.

21. Hesse H \& Hoefgen R (2003). Molecular aspects of methionine biosynthesis. Trends in Plant Science, 8: 259-262.

22. Hesse H, Nikiforova V, Gakiere B \& Hoefgen R (2004). Molecular analysis and control of cysteine biosynthesis: integration of nitrogen and sulfur metabolism. Journal of Experimental Botany, 55: 12831292.

23. Tang GL \& Galili G (2004). Using RNAi to improve plant nutritional value: from mechanism to application. Trends in Biotechnology, 22 : 463-469.

24. Zhu XH \& Galili G (2003). Increased lysine synthesis coupled with a knockout of its catabolism synergistically boosts lysine content and also transregulates the metabolism of other amino acids in Arabidopsis seeds. Plant Cell, 15: 845-853.

25. Curien G, Ravanel S \& Dumas R (2003). A kinetic model of the branch point between the methionine and threonine biosynthesis pathways in Arabidopsis thaliana. European Journal of Biochemistry, 270: 4615-4627.

26. Rognes SE, Lea PJ \& Miflin BJ (1980). S-adenosylmethionine - a novel regulation of aspartate kinase. Nature, 287: 357-359.

27. Rognes SE, Dewaele E, Aas SF, Jacobs M \& Frankard V (2003). Transcriptional and biochemical regulation of a novel Arabidopsis thaliana bifunctional aspartate kinase-homoserine dehydrogenase gene isolated by functional complementation of a yeast hom6 mutant. Plant Molecular Biology, 51: 281-294.

28. Lee SI, Kim HU, Lee YH, Such SC, Lim YP, Lee HY \& Kim HI (2001). Constitutive and seed-specific expression of a maize lysine-feedback-insensitive dihydrodipicolinate synthase gene leads to increased free lysine in rice seeds. Molecular Breeding, 8: 75-84.

29. Fornazier RF, Azevedo RA, Ferreira RR \& Varisi VA (2003). Lysine catabolism: flow, metabolic role and regulation. Brazilian Journal of Plant Physiology, 15: 9-18.

30. Sodek L \& Wilson CM (1970). Incorporation of leucine-C ${ }^{14}$ into protein in the developing of normal and opaque-2 corn. Archives of Biochemistry and Biophysics, 140: 29-38.

31. Arruda P, Kemper EL, Papes F \& Leite A (2000). Regulation of lysine catabolism in higher plants. Trends in Plant Science, 5: 324330.

32. Gaziola SA, Teixeira CMG, Lugli J, Sodek L \& Azevedo RA (1997). The enzymology of lysine catabolism in rice seeds - Isolation, characterization, and regulatory properties of a lysine 2-oxoglutarate reductase saccharopine dehydrogenase bifunctional polypeptide. European Journal of Biochemistry, 247: 364-371.

33. Galili $G$ (2002). New insights into the regulation and functional significance of lysine metabolism in plants. Annual Review of Plant Biology, 53: 27-43.

34. Moulin M, Deleu C \& Larher F (2000). L-Lysine catabolism is osmoregulated at the level of lysine-ketoglutarate reductase and saccharopine dehydrogenase in rapeseed leaf discs. Plant Physiology and Biochemistry, 38: 577-585.

35. Karchi H, Miron D, Ben-Yaacov S \& Galili G (1995). The lysinedependent stimulation of lysine catabolism in tobacco seed requires $\mathrm{Ca}^{2+}$ and protein phosphorylation. Plant Cell, 7: 1963-1970.

36. Gaziola SA, Alessi ES, Guimarães PEO, Damerval C \& Azevedo RA 
(1999). Quality protein maize: a biochemical study of enzymes involved in lysine metabolism. Journal of Agricultural and Food Chemistry, 47: 1268-1275.

37. Kemper EL, Cord-Neto G, Papes F, Martinez-Moraes KC, Leite A \& Arruda P (1999). The role of opaque-2 on the control of lysine degrading activities in developing maize endosperm. Plant Cell, 11: 1981-1994.

38. Gibbon BC, Wang X \& Larkins BA (2002). Proteomic analysis of modified opaque2 maize endosperm. Molecular Biology of the Cell, 13: 258A (Abstract).

39. Ye X, Al-Babili S, Klöti A, Zhang J, Lucca P, Beyer P \& Potrykus I (2000). Engineering the provitamin A (ß-carotene) biosynthetic pathway into (carotenoid-free) rice endosperm. Science, 287: 303-305.

40. Hoa TTC, Al-Babili S, Schaub P, Potrykus I \& Beyer P (2003). Golden indica and japonica rice lines amenable to deregulation. Plant Physiology, 133: 161-169.

41. Garcia AAF \& Souza CL (2002). Phenotypic recurrent selection to improve protein quality in non-opaque maize populations. Scientia Agricola, 59: 743-748.

42. Falco SC (2001). Increasing lysine in corn. Amino Acids, 21: 57-58.

43. Falco SC, Guida T, Locke M, Mauvais J, Sanders C, Ward RT \& Weber P (1995). Transgenic canola and soybean seeds with increased lysine. Bio-Technology, 13: 557-582.

44. Lima STC, Azevedo RA \& Santoro LG (2003). Improved procedures for extraction of lysine 2-oxoglutarate reductase/saccharopine dehydrogenase (LOR/SDH) enzyme from Phaseolus vulgaris cultivars. New Zealand Journal of Crop and Horticultural Science, 31: 261-268.

45. Lima STC, Azevedo RA, Santoro LG, Gaziola SA \& Lea PJ (2003). Isolation of the bifunctional enzyme lysine 2-oxoglutarate reductase-saccharopine dehydrogenase from Phaseolus vulgaris. Amino Acids, 24: 179-186.

46. Azevedo RA, Arana JL \& Arruda P (1990). Biochemical genetics of the interaction of the lysine plus threonine resistant mutant $L \operatorname{tr}^{*} 19$ with opaque-2 maize mutant. Plant Science, 70: 81-90.

47. Lalonde S, Wipf D \& Frommer WB (2004). Transport mechanisms for organic forms of carbon and nitrogen between source and sink. Annual Review of Plant Biology, 55: 341-372.

48. Su YH, Frommer WB \& Ludewig U (2004). Molecular and functional characterization of a family of amino acid transporters from Arabidopsis. Plant Physiology, 136: 3104-3113.
49. Fischer WN, Kwart M, Hummel S \& Frommer WB (1995). Substrate specificity and expression profile of amino acid transporters (AAPs) in Arabidopsis. Journal of Biological Chemistry, 270: 16315-16320.

50. Frommer WB, Hummel S, Unseld M \& Ninnemann O (1995). Seed and vascular expression of a high-affinity transporter for cationic amino acids in Arabidopsis. Proceedings of the National Academy of Sciences, USA, 92: 12036-12040.

51. Arruda P \& Da Silva WJ (1979). Amino acid composition of vascular sap of maize ear peduncle. Phytochemistry, 18: 409-410.

52. Da Silva WJ \& Arruda P (1979). Evidence for the genetic control of lysine in maize endosperm. Phytochemistry, 18: 1803-1805.

53. Damerval C \& Le Guilloux M (1998). Characterization of novel proteins affected by the 02 mutation and expressed during maize endosperm development. Molecular and General Genetics, 257: 354-361.

54. Brennecke K, Souza Neto AJ, Lugli J, Lea PJ \& Azevedo RA (1996). Aspartate kinase in the maize mutants Ask1-LT19 and Opaque-2. Phytochemistry, 41: 707-712.

55. Wang $X$ \& Larkins BA (2001). Genetic analysis of amino acid accumulation in opaque-2 maize endosperm. Plant Physiology, 125: 1766-1777.

56. Azevedo RA, Damerval C, Landry J et al. (2003). Regulation of maize lysine metabolism and endosperm protein synthesis by opaque and floury mutations. European Journal of Biochemistry, 270: 4898-4908.

57. Azevedo RA, Damerval C, Lea PJ et al. (2004). Endosperm protein synthesis and lysine metabolism in distinct opaque maize seed mutants. Functional Plant Biology, 31: 339-348.

58. Azevedo RA, Lea PJ, Damerval C et al. (2004). Regulation of lysine metabolism and endosperm protein synthesis by the opaque- 5 and opaque-7 maize mutations. Journal of Agricultural and Food Chemistry, 52: 4865-4871.

59. Fornazier RF, Gaziola SA, Helm CV, Lea PJ \& Azevedo RA (2005). Isolation and characterization of enzymes involved in lysine catabolism from sorghum seeds. Journal of Agricultural and Food Chemistry, 53: 1791-1798.

60. Helm CV, De Francisco A, Gaziola SA, Fornazier RF, Pompeu GB \& Azevedo RA (2004). Hull-less barley varietes: storage proteins and amino acid distribution in relation to nutritional quality. Food Biotechnology, 18: 327-341. 